\title{
Le maïs ensilage dans le Grand-Ouest français face au changement climatique à l'horizon 2100
}

\author{
François Beauvais $^{* 12}$, Olivier Cantat ${ }^{1}$ et Philippe Madeline ${ }^{2}$ \\ ${ }^{1}$ Normandie Université, UNICAEN, CNRS, LETG, 14000 Caen, France \\ ${ }^{2}$ Normandie Université, UNICAEN, CNRS, ESO, 14000 Caen, France
}

\begin{abstract}
Résumé - Le maïs destiné à l'ensilage est en majeure partie cultivé dans l'Ouest de la France où il contribue à l'alimentation animale d'un territoire spécialisé dans l'élevage bovin. Cet article projette la phénologie de cette plante à l'horizon 2100 ainsi que les aléas qui surviennent au cours des périodes clés de son cycle. Les résultats sont issus d'une modélisation bioclimatique reposant sur les données du modèle régional ALADIN-Climat (DRIAS 2020) pour les scénarios RCP 2.6 et RCP 8.5. Face au changement climatique envisagé pour le futur, l'augmentation des températures réduirait considérablement la durée du cycle du maïs. Cette plante pourrait alors être exposée à des stress hydriques importants pénalisant la photosynthèse. La floraison rencontrerait des excès thermiques de façon récurrente pour la fertilité de la plante. Ainsi, il conviendrait de développer des variétés plus tolérantes à ces divers aléas, en parallèle des alternatives déjà initiées concernant par exemple la luzerne et le sorgho.
\end{abstract}

Mots-clés : changement climatique, maïs ensilage, région Grand-Ouest, France, bioclimatologie.

\begin{abstract}
Maize silage in the French Grand-Ouest and climate change by 2100. Silage maize is mostly grown in western France, where it contributes to animal feed in an area specialised in cattle breeding. This article projects the phenology of this plant to 2100 , as well as the hazards that occur during the key periods of its cycle. The results are based on bioclimatic modelling using data from the regional ALADIN-Climat (DRIAS 2020) model for the RCP 2.6 and RCP 8.5 scenarios. In view of future climate change, the increase in temperature would considerably reduce the cycle length of maize. This plant could then be exposed to major water stresses that would penalise photosynthesis. Flowering would encounter recurrent thermal excesses for the fertility of the plant. Thus, it would be advisable to develop varieties that are more tolerant to these various hazards, in parallel with the alternatives already initiated for alfalfa and sorghum, for example.
\end{abstract}

Keywords: climate change, silage maize, Grand-Ouest region, France, bioclimatology.

* $\overline{\text { Auteur de correspondance }}$ : francois.beauvais@unicaen.fr

\section{Introduction}

En France, le maïs est cultivé sur près de 2,9 millions d'hectares (Registre Parcellaire Graphique 2016). Parmi ces surfaces, $44 \%$ sont destinées au maïs ensilage pour le bétail et le reste pour l'exploitation des grains pour l'amidonnerie. À lui seul, le Grand Ouest français concentre près de $60 \%$ des surfaces et des productions nationales de maïs fourrage. L'ensemble aérien de la plante (tige, feuilles et grains) est récolté et ensilé pour l'alimentation des bovins qui caractérisent cette région spécialisée dans l'élevage pour le lait et la viande. Cette technique s'est développée à partir des années 1970 grâce à l'introduction de variétés précoces, adaptées à l'offre plus modeste de chaleur dans le nord-ouest du pays. A la fin des années 1970, les surfaces atteignent le million d'hectares, pour se stabiliser depuis la fin $\mathrm{du} \mathrm{XX}^{\mathrm{e}}$ siècle autour de 1,4 à 1,5 million d'ha. Toutefois, elles varient selon les conditions climatiques, très variables d'une année à l'autre en domaine océanique tempéré. Lorsqu'elles sont défavorables et limitent le rendement du maïs grain, celui-ci peut d'ailleurs 
être récolté plus tôt dans la saison pour le fourrage (Carpentier et Cabon, 2011).

Comme l'ensemble de la planète, le nord-ouest de la France est concerné par le changement climatique. Ce constat qui inquiète la profession agricole et l'ensemble des acteurs socioéconomiques est mis en avant notamment dans les résultats pour le Grand Ouest du programme CLIMASTER (2008-2011). Au début de la décennie 2010, Dubreuil et al. (2013a) font le constat que le réchauffement est homogène sur l'ensemble de cette région de l'hexagone, avec une hausse de la température moyenne d'un degré en seulement 50 ans. Du côté des précipitations, les auteurs précisent qu'elles sont sujettes à une très forte variabilité spatiale et temporelle et ne signalent pas d'évolution statistiquement significative au pas de temps annuel. Néanmoins, à l'échelle saisonnière, les étés semblent plus secs (principalement en août) et les hivers plus arrosés. Ce changement climatique devrait se poursuivre dans les prochaines décennies, avec une accélération de la hausse des températures après 2050 et une réduction des précipitations totales annuelles d'environ $20 \%$ (Dubreuil et al., 2013b). Les sécheresses, plus récurrentes et plus prononcées telles qu'en témoignent les recherches menées en Bretagne et en Normandie (Cantat et al., 2009 ; Cantat et al., 2010 ; Le Gouée et al., 2010a ; Le Gouée et al., 2010b ; Lamy et al., 2013 ; Lamy et Dubreuil, 2013 ; Dubreuil et al., 2018 ; Beauvais et al., 2019) sont attendues dans une région déjà soumise à ce risque dès le printemps (Dubreuil, 1994). Les conséquences prévisibles pour les productions végétales sont principalement induites par la précocité de la phénologie et les stress hydriques et thermiques. Ces tendances sont confirmées par les données issues des dernières simulations du modèle climatique régional ALADIN-Climat mobilisées dans le cadre de cet article.

Pour s'approcher au plus près des conditions vécues par la plante, nous proposons de faire appel à une approche bioclimatique déjà mobilisée pour la culture du blé tendre et du lin fibre de printemps en Normandie (Beauvais et al., 2019a, 2019b, 2020). Le recours à des indicateurs climatiques calculés sur les périodes phénologiques sensibles de la plante sont plus précis que ceux calés sur des dates invariantes (Holzkamper et al., 2013). Cette méthode qui s'adapte au déroulement réel de la vie de la plante a déjà été validée dans plusieurs recherches récentes (Gate et al., 2008 ; Gate, 2009 ; Mkhabela et al., 2010 ; Gouache et al., 2012 ; Holzkämper et al., 2011, 2013, 2015 ; Trnka et al., 2014 ; Caubel et al., 2015, 2018 ; Beauvais et al., 2019a, 2019b, 2020). Elle intervient en complément des recherches fondées sur les modèles de cultures. Cette méthode n'a pas encore été développée pour le maïs fourrager dans le Grand Ouest de la France.

Dans cet article, trois espaces de culture représentatifs de la culture du maïs dans le Grand Ouest de la France ont été choisis (figure 1), exprimant les nuances de conditions climatiques liées aux dégradations de l'océanité (d'ouest en est) et l'augmentation de la méridionalité (du nord au sud). Il s'agit de la pointe de la Bretagne (fortement océanisée) avec un point de grille proche de la commune de Milizac (A) ; du Pays de Rennes (océanique d'abri) avec un point de grille proche de la commune de Montauban-de-Bretagne (B) ; du Val d'Anjou (océanique adouci) avec un point de grille proche de la commune de Baugé-en-Anjou (C).

\section{Données et méthode}

\subsection{Les données SAFRAN et du modèle climatique régional ALADIN-Climat}

Les données de la période observée (1976-2005) sont celles des réanalyses SAFRAN (QuintanaSegui et al., 2008 ; Soubeyroux et al., 2008 ; Vidal et al., 2010). SAFRAN est un système d'analyse qui combine les observations horaires de surface avec celles des modèles météorologiques. Les paramètres sont analysés par pas de $300 \mathrm{~m}$ d'altitude et interpolées sur une grille horizontale d'une résolution spatiale de 8 x $\quad 8 \quad \mathrm{~km}$ (http://www.umr-cnrm.fr/). Elles sont utiles pour comparer les données du scénario historique des simulations climatiques sur la période de référence 1976-2005 puisque les points de grille sont équivalents. 


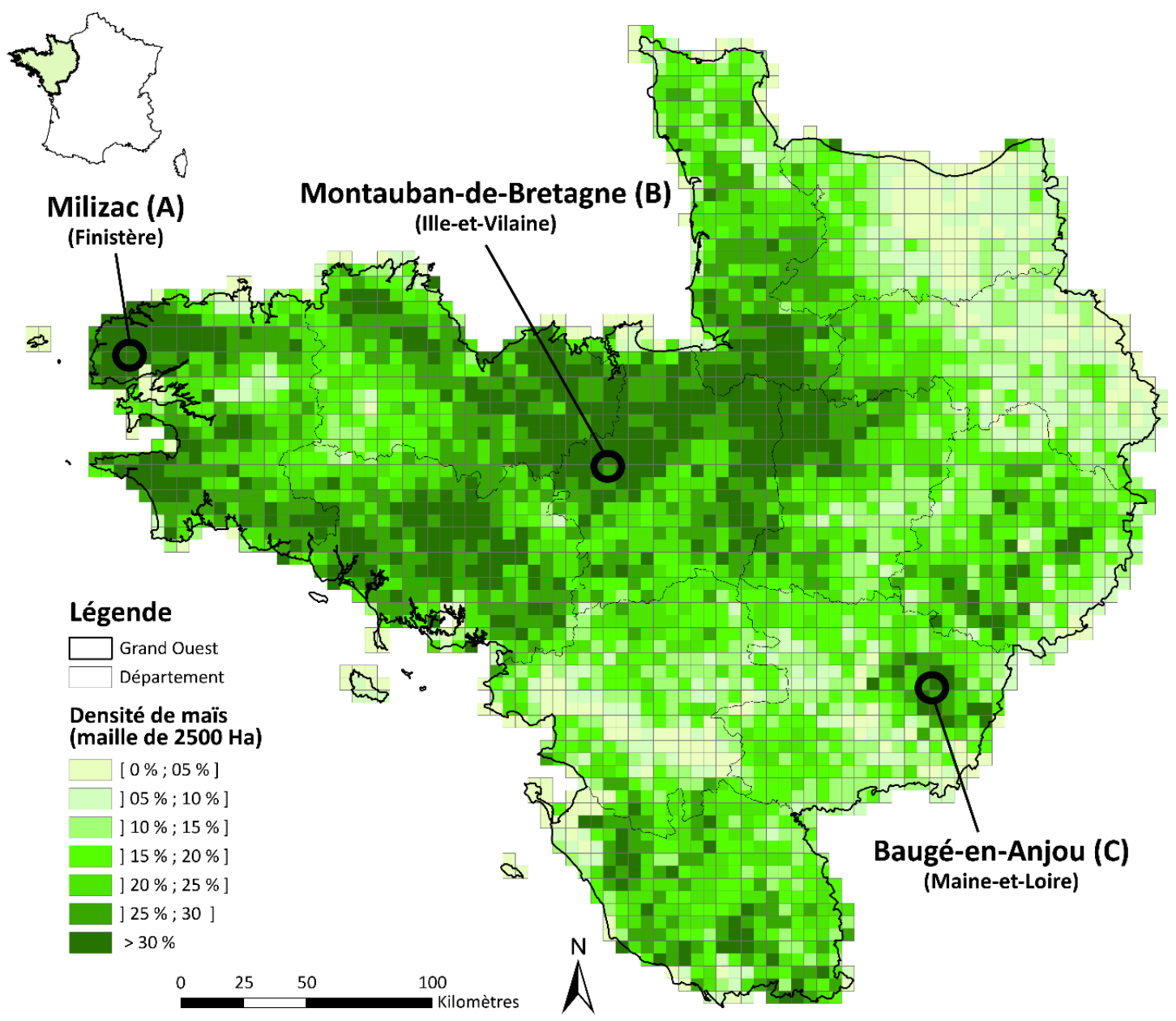

Diagrammes ombrothermiques pour la période de référence 1976-2005 SAFRAN

A.

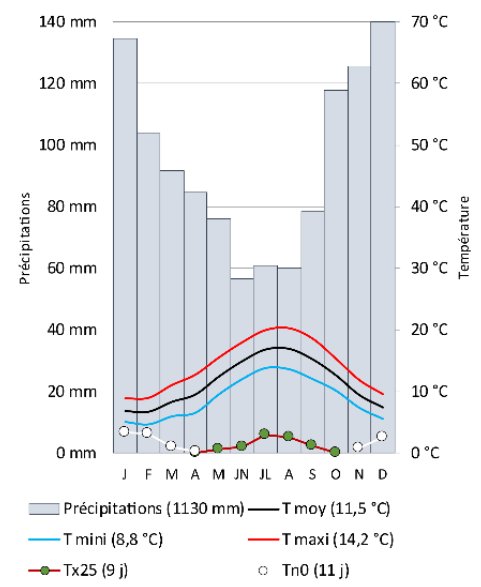

B.

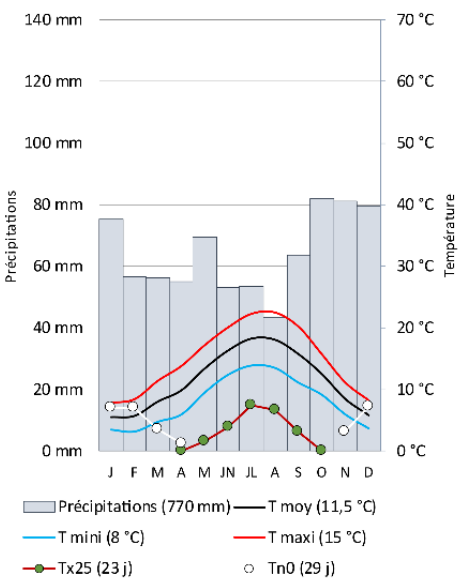

C.

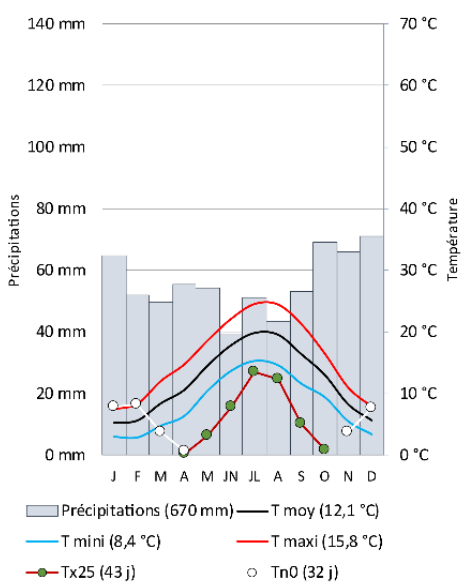

Données : RPG 2016 de l'ASP ; Bd GEOFLA 2016 de I'IGN ; modèle SAFRAN Météo-France.

$\mathrm{Nb}$ : Les mailles SAFRAN sélectionnées sont celles qui se juxtaposent avec les communes présentées sur la carte.

Figure 1. Densité de maïs dans le Grand-Ouest Français et diagramme ombrothermique des sites retenus. Maize density in the French Grand-Ouest and umbrothermal diagram of the selected sites.

Les données pour la période future sont extraites du couple GCM/RCM (avec CNRM-CM5 et ALADIN63_CNRM-CM5) disponible sur le site www.drias-climat.fr (Soubeyroux et al., 2020). Les variables sélectionnées au pas de temps journalier sont les températures minimales et maximales, les 
précipitations totales, le rayonnement solaire, l'humidité spécifique et le rayonnement visible incident à la surface. Les biais du modèle sont corrigés à partir de la méthode ADAMONT (Verfaillie et al., 2017). C'est-à-dire que la distribution des données simulées est comparée à celle des données observées (réanalyse SAFRAN) sur la période 1976-2005 à partir d'une climatologie saisonnière et par types de temps. Confronté à l'observation, le modèle ALADIN63_CNRM-CM5 simule une température moyenne proche de l'observation $\left(-0,1^{\circ} \mathrm{C}\right.$ pour les trois points de grille retenus) et une légère sous-estimation pour les précipitations annuelles (-6\% à Milizac, $-5 \%$ à Montauban-de-Bretagne et à Baugé-en-Anjou). Outre la fiabilité de la modélisation par rapport à l'observation, le choix d'un seul modèle se justifie par le fait que cet article s'articule autour de l'intérêt de l'approche phénoclimatique pour l'étude d'une culture de printemps et non pas d'une analyse multimodèles, comme évoquée dans la partie «discussion». Les projections utilisées couvrent l'ensemble des futurs possibles : elles sont issues du scénario bas (ou «optimiste») RCP 2.6 et du scénario élevé (ou « pessimiste ») RCP 8.5 du GIEC (Van Vuuren et al., 2011) pour l'horizon lointain (2071-2100) afin d'exprimer le changement du bilan radiatif au sommet de la troposphère.

\subsection{Méthode d'analyse phénoclimatique}

La durée des périodes phénologiques et les dates d'apparition des stades sont calculées à partir du concept de degrés-jours (Bonhomme, 2000). La somme de température pour la croissance du maïs s'effectue en base $6^{\circ} \mathrm{C}$, écrêtée à $30^{\circ} \mathrm{C}$. Ainsi, les températures inférieures à $6^{\circ} \mathrm{C}$ comptent pour nulles et celles supérieures à $30^{\circ} \mathrm{C}$ sont considérées à hauteur de ce seuil. Les stades simulés sont les suivants: émergence, 3 feuilles, 6 feuilles, 14 feuilles, floraison et maturité $32 \%$ MS (Matière sèche $)^{1}$. Les sommes de températures retenues correspondent aux gammes de valeurs présentées

\footnotetext{
${ }^{1}$ Le maïs fourrager est récolté à $32 \%$ de Matière sèche pour des raisons de conservation et d'ingestion pour l'animal (https://www.semencesdefrance.com/dossier/mais-quand-recolter/). Le maïs grain quant à lui est récolté pour un taux d'humidité de 20 à $25 \%$.

2 https://www.arvalis-infos.fr/ma-s-peut-on-deja-commencerles-semis--@/view-32196-arvarticle.html
}

par Arvalis L'Institut du Végétal (2021) pour une variété précoce et une variété tardive. La première nécessite 870 et $1480^{\circ} \mathrm{CJ}$ pour la floraison et la maturité $32 \%$ MS. Les besoins en chaleur de la seconde s'élèvent à 1050 et $1740^{\circ} \mathrm{CJ}$. Il s'agit des gammes de valeurs présentées par Arvalis L'Institut du Végétal (2021). La date de semis pour le calcul de cette phénologie s'établit pour un ensemencement le $1^{\text {er }}$ mai de chaque année. On considère alors que l'exploitant tient compte du risque d'exposition au froid et à l'humidité du mois d'avril. En effet, les semis trop précoces sont pénalisants pour le maïs fourrage, limitant les surfaces foliaires et engendrant des plantes plus courtes $^{2}$.

Le maïs est sensible à plusieurs aléas climatiques, notamment recensés par Holzkamper et al. (2013), Caubel et al. (2018) ou encore par les sites internet des structures agricoles ${ }^{3}$. Les années chaudes réduisent la durée du cycle, engendrant une diminution de l'accumulation de biomasse et des rendements. Le gel interrompt la photosynthèse, bloquant ainsi les transferts de la tige vers les feuilles. Les gelées inférieures à $-1^{\circ} \mathrm{C}$ peuvent stresser la plante, voire engendrer sa mortalité dans le cas de températures plus basses. Les températures très élevées sont pénalisantes pour la pollinisation et peuvent, lorsqu'elles sont associées à un déficit hydrique, favoriser l'enroulement des feuilles. D'après Hawkins et al. (2013), une réduction significative $\mathrm{du}$ rendement s'observe quand la température maximale excède $32^{\circ} \mathrm{C}$, notamment lorsqu'elle coïncide avec la floraison ${ }^{4}$. La photosynthèse et la croissance de la plante peuvent être impactées par un déficit hydrique et de rayonnement solaire global. Pour exprimer la satisfaction en eau de la plante, nous avons mobilisé le confort hydrique, variant de 0 (stress hydrique exacerbé) à 1 (conditions optimales), correspondant au rapport ETR/ETM (évapotranspiration réelle et évapotranspiration maximale de la culture). Enfin, les excès d'eau en surface du sol peuvent engendrer,

\footnotetext{
3 www.fiches.arvalis-infos.fr/liste fiches.php?fiche=acc\&type=AM www.dekalb.fr/mais-grain/conseils-pour-planter-et-cultiver-lemais/problemes-climatiques-et-nutriments

${ }^{4}$ Nous avons calculé l'occurrence de cet aléa sur les $100^{\circ} \mathrm{CJ}$ entourant ce stade phénologique afin de considérer la période «proche floraison ».
} 
notamment au début du cycle, une asphyxie de la plante qui ne peut plus échanger avec l'atmosphère. Nous retenons alors ces aléas sous la forme d'indicateurs phénoclimatiques. On calcule leur occurrence sur les phases sensibles du cycle de la plante à partir de fonctions itératives.

Les conditions bio-pédoclimatiques sont déterminées à l'aide d'un bilan hydrique journalier à décroissance logarithmique de la réserve utile, selon la méthode de Thornthwaite et Mather (1955), permettant de tenir compte de la difficulté progressive de la plante à mobiliser l'eau du sol à mesure que la réserve se vide. Les besoins en eau de la plante sont traduits par des coefficients culturaux (Doorenbos et Pruitt, 1975) qui varient au cours du cycle de la plante, comme exposé par Allen et al. (1998). Ils sont multipliés à l'ETP (évapotranspiration potentielle) pour déterminer l'ETM de la plante. La formule utilisée pour le calcul de l'ETP est celle de Turc $^{5}$ (Turc, 1961) tenant compte de la température, du rayonnement solaire global et de l'humidité relative de l'air.

Le Grand Ouest dispose de sols aux capacités de rétention en eau variées. Ainsi, nous avons retenu pour chaque site l'hypothèse de deux RUM (Réservoir Utilisable Maximal) traduisant la diversité possible des sols, avec $200 \mathrm{~mm}$ (sol épais que le maïs peut valoriser en raison d'un enracinement de $90 \mathrm{~cm}$ ) et $50 \mathrm{~mm}$ (sol mince).

\section{Résultats}

\subsection{Réduction de la durée du cycle et faisabilité d'une variété tardive}

Sur la période de référence 1976-2005 (figure 2), la floraison de la variété précoce intervient le 6 août à Milizac (A), le 29 juillet à Montauban-deBretagne (B) et le 19 juillet à Baugé-en-Anjou (C). Dans le cas du scénario RCP 2.6, elle apparaîtrait 8 à 9 jours plus tôt sur les trois sites. Avec le scénario RCP 8.5, l'anticipation est en revanche plus prononcée, respectivement de 22, 19 et 16 jours pour Milizac (A), Montauban-de-Bretagne (B) et Baugé-en-Anjou (C). La maturité à $32 \%$ MS est quant à elle atteinte le 7 octobre à Milizac (A), le 20 septembre à Montauban-de-Bretagne $(\mathrm{B})$ et le $1^{\text {er }}$ septembre à Baugé-en-Anjou (C). Dans le cas du scénario RCP 2.6, la récolte pourrait débuter respectivement 20, 17 et 13 jours plus tôt. Sans réduction des émissions de gaz à effet de serre (scénario RCP 8.5), l'offre de température est telle que l'ensilage serait anticipé respectivement de 43, 35 et 26 jours. Par conséquent, le cycle se clôturerait à Milizac (A) à une date équivalente à celle observée en période de référence à Baugé-en-Anjou (C), ce qui exprime l'ampleur des conséquences du réchauffement attendu si aucune politique climatique internationale n'est rapidement mise en place.

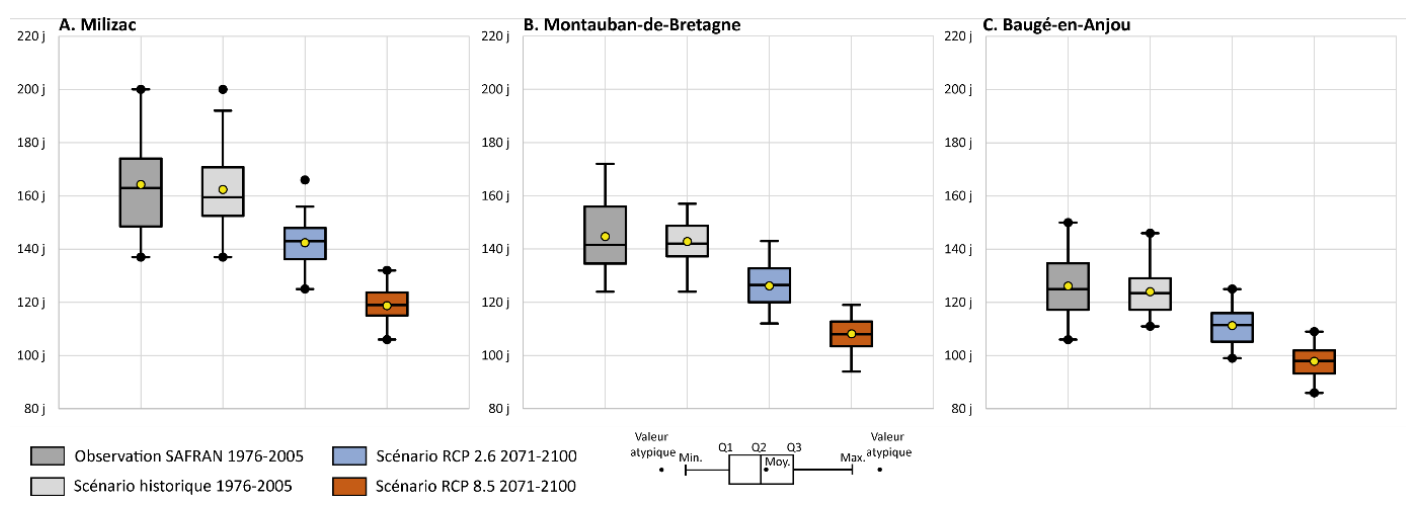

Figure 2 : Durée du cycle (du semis à la maturité $32 \%$ MS) du maïs fourrager pour la période de référence 1976-2005 et lointaine 2071-2100 selon deux scénarios RCP à Milizac (à gauche), Montauban-de-Bretagne (au centre) et Baugéen-Anjou (à droite). Cycle length (from sowing to maturity 32\% DM - Dry Mater) of silage maize for the reference period 1976-2005 and 2071-2100 according to two RCP scenarios in Milizac (left), Montauban-de-Bretagne (center) and Baugé-en-Anjou (right).

5 L'ETP Turc est également calculée pour les données observées. En effet, bien que nous disposions de l'ETP Penman avec les réanalyses SAFRAN, la demande climatique, pour être comparable, doit être estimée de la même façon que pour les projections climatiques. 


\subsection{Augmentation du déficit hydrique et dégradation du confort hydrique}

Le maïs déroule son cycle en périodes printanière et estivale, soit à un moment de l'année durant lequel la demande bioclimatique (l'évapotranspiration) est supérieure à l'offre (les précipitations). Le début du cycle, jusqu'au stade 6 feuilles, n'est que peu impacté par un déficit hydrique de quelques millimètres. Le maïs profite de la réserve en eau du sol acquise lors de la période automnale et hivernale. Aussi, la demande de la plante est moins importante en début de cycle, contrairement aux stades suivants qui ont le désavantage de s'exprimer lorsque la réserve commence à se vider (figure 3). Sur les sols épais du secteur de Milizac (A), le manque d'eau (pour un maïs non irrigué) est d'environ $60 \mathrm{~mm}$ sur la totalité du cycle de la plante en période historique. A Montauban-de-Bretagne (B), il est un peu plus prononcé avec environ $80 \mathrm{~mm}$. À Baugé-en-Anjou (C), il est supérieur à $100 \mathrm{~mm}$. Dans le cas d'un sol mince, le manque d'eau est deux fois plus important. Derrière ces valeurs moyennes peuvent se cacher des situations extrêmes telles que la sécheresse de 1976. Cette année-là, le manque d'eau pour le maïs cultivé sur sol épais est supérieur à celui observé en moyenne sur sol mince. À Milizac (A) et à Montauban-de-Bretagne (B), le déficit hydrique atteint 180 à $190 \mathrm{~mm}$, tandis qu'il s'éleva à $240 \mathrm{~mm}$ à Baugé-en-Anjou (C). Pour le sol mince, les conséquences hydriques de cette situation extrême furent encore plus prononcées : 260 à $270 \mathrm{~mm}$ pour Milizac (A) et Montauban-deBretagne (B), $310 \mathrm{~mm}$ pour Baugé-en-Anjou (C). La comparaison entre ces trois sites en période historique montre que le manque d'eau pour le maïs est plus prononcé à mesure que l'on se dirige vers l'est. Le cycle pourtant plus court n'étant pas en mesure de compenser les conditions plus sèches. Ce constat se retrouve dans l'interprétation du confort hydrique, en témoignent les indices d'environ 0,8 et 0,6 respectivement pour le cycle du maïs cultivé sur des sols épais et mince à Milizac (A), alors même qu'il s'agit du site le plus océanique (tableau 1). À Baugé-en-Anjou (C), l'inconfort de la plante est plus prononcé puisque ces indices sont de seulement 0,6 et 0,4 pour les deux types de sol dans le cas de la période « 14 feuilles - floraison ».

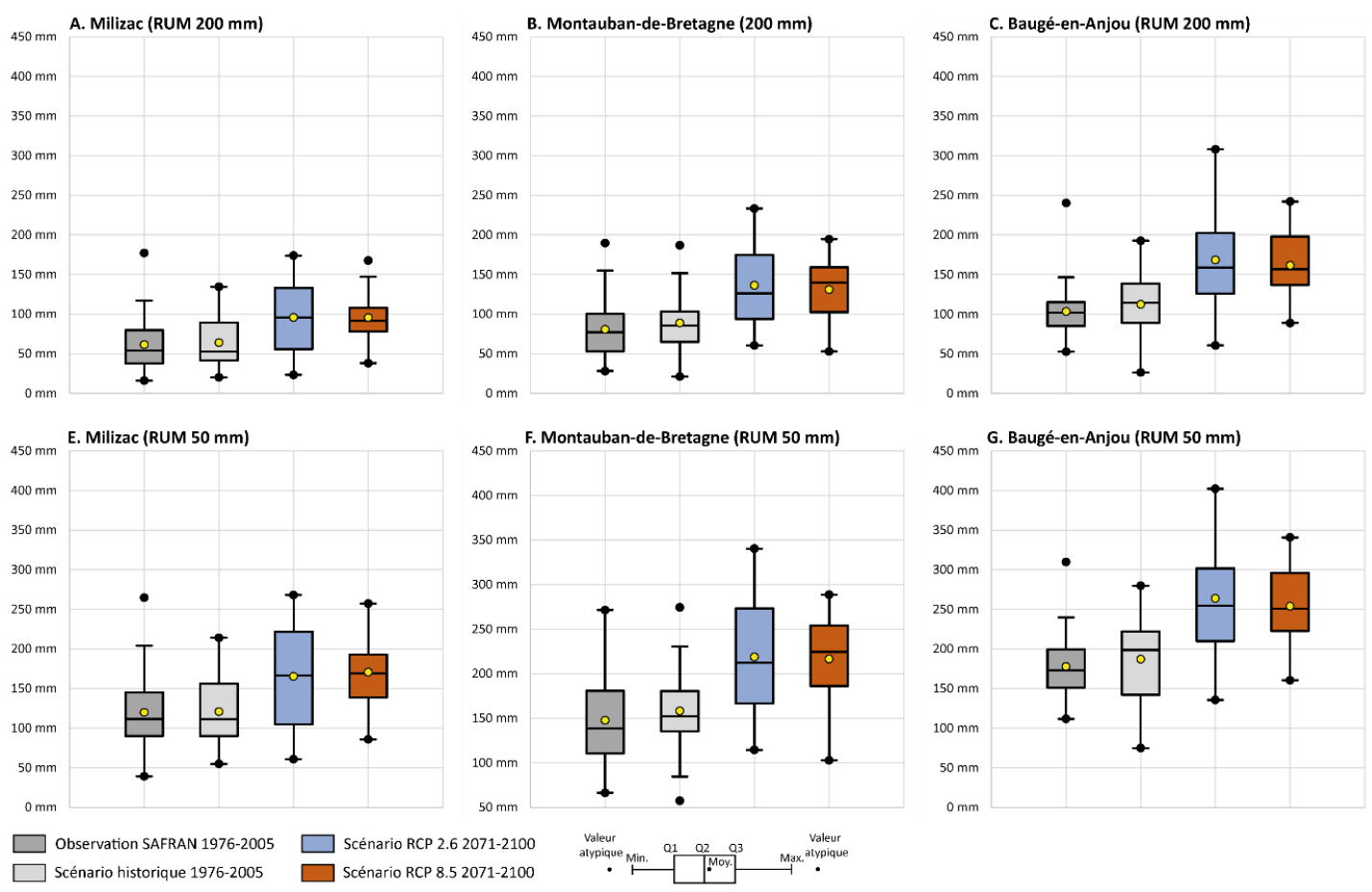

Figure 3. Déficit hydrique cumulé sur la totalité du cycle du maïs fourrager pour un RUM $200 \mathrm{~mm}$ (en haut) et $50 \mathrm{~mm}$ (en bas) pour la période de référence 1976-2005 et lointaine 2071-2100 selon deux scénarios RCP à Milizac (à gauche), Montauban-de-Bretagne (au centre) et Baugé-en-Anjou (à droite). Cumulative water deficit over the entire cycle of forage maize for a RUM of $200 \mathrm{~mm}$ (top) and $50 \mathrm{~mm}$ (bottom) for the reference period 1976-2005 and 2071-2100 according to two RCP scenarios in Milizac (left), Montauban-de-Bretagne (center) and Baugé-en-Anjou (right). 
Tableau 1. Confort hydrique du maïs fourrager au cours des périodes phénologiques 14 feuilles - floraison et floraison - maturité 32 \% MS pour la période de référence 1976-2005 et lointaine 2071-2100 selon deux scénarios RCP à Milizac, Montauban-de-Bretagne et Baugé-en-Anjou. Water comfort of silage maize during the phenological periods 14 leaves - flowering and flowering - maturity 32\% DM for the reference period 1976-2005 and 2071-2100 according to two RCP scenarios in Milizac, Montauban-de-Bretagne and Baugé-en-Anjou.

\begin{tabular}{|c|c|c|c|c|c|}
\hline \multirow{10}{*}{ 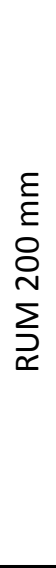 } & Période phénologique & $\begin{array}{c}\text { Observation } \\
\text { SAFRAN } \\
1976-2005\end{array}$ & $\begin{array}{c}\text { Scénario } \\
\text { historique } \\
1976-2005\end{array}$ & \begin{tabular}{|c} 
Scénario RCP 2.6 \\
$2071-2100$ \\
\end{tabular} & \begin{tabular}{|c} 
Scénario RCP 8.5 \\
$2071-2100$
\end{tabular} \\
\hline & \multicolumn{5}{|c|}{ Milizac } \\
\hline & 14 feuilles - floraison & 0,8 & 0,8 & 0,7 & 0,7 \\
\hline & floraison - maturité $32 \% \mathrm{MS}$ & 0,8 & 0,8 & 0,7 & 0,7 \\
\hline & \multicolumn{5}{|c|}{ Montauban-de-Bretagne } \\
\hline & 14 feuilles - floraison & 0,7 & 0,7 & 0,5 & 0,5 \\
\hline & floraison - maturité $32 \% \mathrm{MS}$ & 0,8 & 0,8 & 0,6 & 0,6 \\
\hline & \multicolumn{5}{|c|}{ Baugé-en-Anjou } \\
\hline & 14 feuilles - floraison & 0,7 & 0,7 & 0,6 & 0,6 \\
\hline & floraison - maturité $32 \% \mathrm{MS}$ & 0,6 & 0,6 & 0,5 & 0,5 \\
\hline
\end{tabular}

\begin{tabular}{|c|c|c|c|c|c|}
\hline \multirow{10}{*}{ 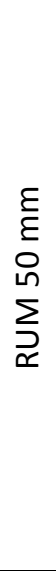 } & Période phénologique & $\begin{array}{c}\text { Observation } \\
\text { SAFRAN } \\
\text { 1976-2005 } \\
\end{array}$ & $\begin{array}{c}\text { Scénario } \\
\text { historique } \\
1976-2005 \\
\end{array}$ & \begin{tabular}{|c|} 
Scénario RCP 2.6 \\
$2071-2100$ \\
\end{tabular} & \begin{tabular}{|c} 
Scénario RCP 8.5 \\
$2071-2100$ \\
\end{tabular} \\
\hline & \multicolumn{5}{|c|}{ Milizac } \\
\hline & 14 feuilles - floraison & 0,6 & 0,6 & 0,5 & 0,5 \\
\hline & floraison - maturité $32 \% \mathrm{MS}$ & 0,7 & 0,7 & 0,6 & 0,5 \\
\hline & \multicolumn{5}{|c|}{ Montauban-de-Bretagne } \\
\hline & 14 feuilles - floraison & 0,5 & 0,5 & 0,4 & 0,4 \\
\hline & floraison - maturité $32 \% \mathrm{MS}$ & 0,7 & 0,7 & 0,5 & 0,3 \\
\hline & \multicolumn{5}{|c|}{ Baugé-en-Anjou } \\
\hline & 14 feuilles - floraison & 0,4 & 0,4 & 0,3 & 0,3 \\
\hline & floraison - maturité $32 \% \mathrm{MS}$ & 0,5 & 0,5 & 0,3 & 0,3 \\
\hline
\end{tabular}

D'ici 2100, le manque d'eau pour la plante serait équivalent entre les scénarios RCP 2.6 et 8.5. Il serait respectivement pour les sols épais et minces d'environ 95 et $165 \mathrm{~mm}$ à Milizac $(\mathrm{A}) ; 130$ et 220 mm à Montauban-de-Bretagne (B); et 170 et 260 mm à Baugé-en-Anjou $(\mathrm{C})$. Autrement dit, le déficit hydrique augmenterait d'environ $50 \%$ par rapport à la période de référence. Si les valeurs de déficit hydrique sont très voisines entre les deux scénarios «optimiste» et «pessimiste», c'est que la réduction de la durée du cycle dans le cas du réchauffement plus fort associé au RCP 8.5 permettrait de diminuer le temps d'exposition à la sécheresse.

À Montauban-de-Bretagne (B) et Baugé-enAnjou (C), les années les plus défavorables du scénario RCP 2.6 verraient le maïs cumuler un déficit hydrique supérieur à celui de la sécheresse de 1976 en raison d'une plus grande variabilité interannuelle. Avec le scénario RCP 8.5, le seuil de 1976 n'est pas franchi. Néanmoins, les conditions seraient tout de même plus sèches, en témoigne la distribution statistique illustrée en figure 3 . Le déficit hydrique médian serait supérieur au $3^{\text {ème }}$ quartile de la période de référence.

Cette évolution à la hausse du manque d'eau pour la plante se traduit par une dégradation du confort hydrique avec les deux scénarios RCP (tableau 1). Le maïs cultivé sur sol épais resterait relativement épargné à Milizac (A ; indice de 0,7). En revanche, ce rapport ETR/ETM serait seulement de 0,5 à Montauban-de-Bretagne (B) et Baugé-enAnjou (C), respectivement pour les phases « 14 feuilles - floraison » et « floraison - maturité $32 \%$ MS ». Le fort RUM ne permet pas alors de compenser la sécheresse estivale. La situation serait 
donc naturellement beaucoup plus critique pour le maïs déroulant son cycle sur les petits sols, et ce même dans le climat naturellement moins contraignant de Milizac (A) avec un confort hydrique de 0,5. Tandis qu'à Baugé-en-Anjou (C), la situation s'aggraverait encore davantage (indice de 0,3$)$.

\subsection{Augmentation des excès thermiques lors de la floraison}

En semant le maïs le $1^{\text {er }}$ mai, aucune gelée inférieure à $-1^{\circ} \mathrm{C}$ n'est relevée. Avec le réchauffement d'ici la fin du siècle, ce risque reste écarté. Il en va de même pour la fin du cycle qui s'effectue au début de l'automne. Les stress thermiques liés à des températures supérieures à $32^{\circ} \mathrm{C}$ autour de la floraison sont rares en période de référence à
Milizac (A) et à Montauban-de-Bretagne (B) (tableau 2). En revanche, ces épisodes de forte chaleur (au moins un jour) sont observés 9 années sur 30 à Baugé-en-Anjou (C) et constituent alors dans ce cas un facteur limitant. Cet aléa pourrait être plus récurrent d'ici la fin du $21^{\mathrm{e}}$ siècle. L'occurrence s'élève à $8 / 30$ années à Milizac (A) et $11 / 30$ années à Montauban-de-Bretagne (B) et à Baugé (C). Avec le scénario RCP 8.5, seulement 3 années sont concernées à Milizac (A). À Montauban-de-Bretagne (B) et à Baugé-en-Anjou (C), 12 et 19 années seraient pénalisées. Pour ces sites les plus orientaux, 5 à 7 jours peuvent être observés autour de la floraison pour l'année la plus extrême. Autrement dit, presque la totalité des jours entourant la floraison - phase cruciale pour la continuité du cycle de la plante - pourrait s'effectuer en condition de stress thermique.

Tableau 2 : Nombre d'années (sur 30 ans) d'exposition du maïs fourrager à au moins une occurrence de température maximale supérieure ou égale à $32^{\circ} \mathrm{C}$ sur la période proche floraison et occurrence extrême associée (en termes de jours) pour la période de référence 1976-2005 et lointaine 2071-2100 selon deux scénarios RCP à Milizac, Montaubande-Bretagne et Baugé-en-Anjou. Number of years (over 30 years) of exposure of silage maize to at least one occurrence of maximum temperature greater than or equal to $32^{\circ} \mathrm{C}$ during the near-flowering period and associated extreme occurrence (in days) for the 1976-2005 reference period and the 2071-2100 reference period according to two RCP scenarios at Milizac, Montauban-de-Bretagne and Baugé-en-Anjou.

\begin{tabular}{|c|c|c|c|}
\cline { 2 - 4 } \multicolumn{1}{c|}{} & Milizac & $\begin{array}{c}\text { Montauban-de- } \\
\text { Bretagne }\end{array}$ & Baugé-en-Anjou \\
\hline Observation SAFRAN 1976-2005 & $\begin{array}{c}01 / 30 \mathrm{a} \\
(\max : 1 \mathrm{j})\end{array}$ & $\begin{array}{c}03 / 30 \mathrm{a} \\
(\max : 1 \mathrm{j})\end{array}$ & $\begin{array}{c}09 / 30 \mathrm{a} \\
(\max : 3 \mathrm{j})\end{array}$ \\
\hline Scénario historique1976-2005 & $\begin{array}{c}01 / 30 \mathrm{a} \\
(\max : 2 \mathrm{j})\end{array}$ & $\begin{array}{c}03 / 30 \mathrm{a} \\
(\max : 1 \mathrm{j})\end{array}$ & $\begin{array}{c}09 / 30 \mathrm{a} \\
(\max : 3 \mathrm{j})\end{array}$ \\
\hline Scénario RCP 2.6 2071-2100 & $\begin{array}{c}08 / 30 \mathrm{a} \\
(\max : 3 \mathrm{j})\end{array}$ & $\begin{array}{c}11 / 30 \mathrm{a} \\
(\max : 5 \mathrm{j})\end{array}$ & $\begin{array}{c}11 / 30 \mathrm{a} \\
(\max : 6 \mathrm{j})\end{array}$ \\
\hline Scénario RCP 8.5 2071-2100 & $\begin{array}{c}03 / 30 \mathrm{a} \\
(\max : 3 \mathrm{j})\end{array}$ & $\begin{array}{c}12 / 30 \mathrm{a} \\
(\max : 5 \mathrm{j})\end{array}$ & $\begin{array}{c}19 / 30 \mathrm{a} \\
(\max : 7 \mathrm{j})\end{array}$ \\
\hline
\end{tabular}

\subsection{Stabilisation du cumul de rayonnement capté par la plante}

D'ici 2100, la réduction de la durée des phases phénologiques laisserait supposer une exposition moins importante au cumul de rayonnement solaire. Mais ce serait sans compter sur l'intensité plus importante de ce paramètre qui permettrait in fine à la plante de profiter d'un cumul équivalent à la période historique. Sur la phase phénologique 14 feuilles-floraison, le rayonnement cumulé augmenterait de l'ordre de 7 à $13 \%$ selon les sites pour le scénario RCP 2.6 (figure 4). Pour le scénario RCP 8.5 , il n'y a pas d'évolution. Sur la dernière partie du cycle, la floraison-maturité $32 \% \mathrm{MS}$, ce cumul reste globalement inchangé, excepté à Baugé-enAnjou $(\mathrm{C})$ où la hausse est de l'ordre de $10 \%$. À Milizac (A), il conviendra de rester vigilant à des années durant lesquelles ce cumul pourrait s'avérer faible en raison d'une réduction importante de la durée de cette dernière phase du cycle par rapport à la période de référence. 


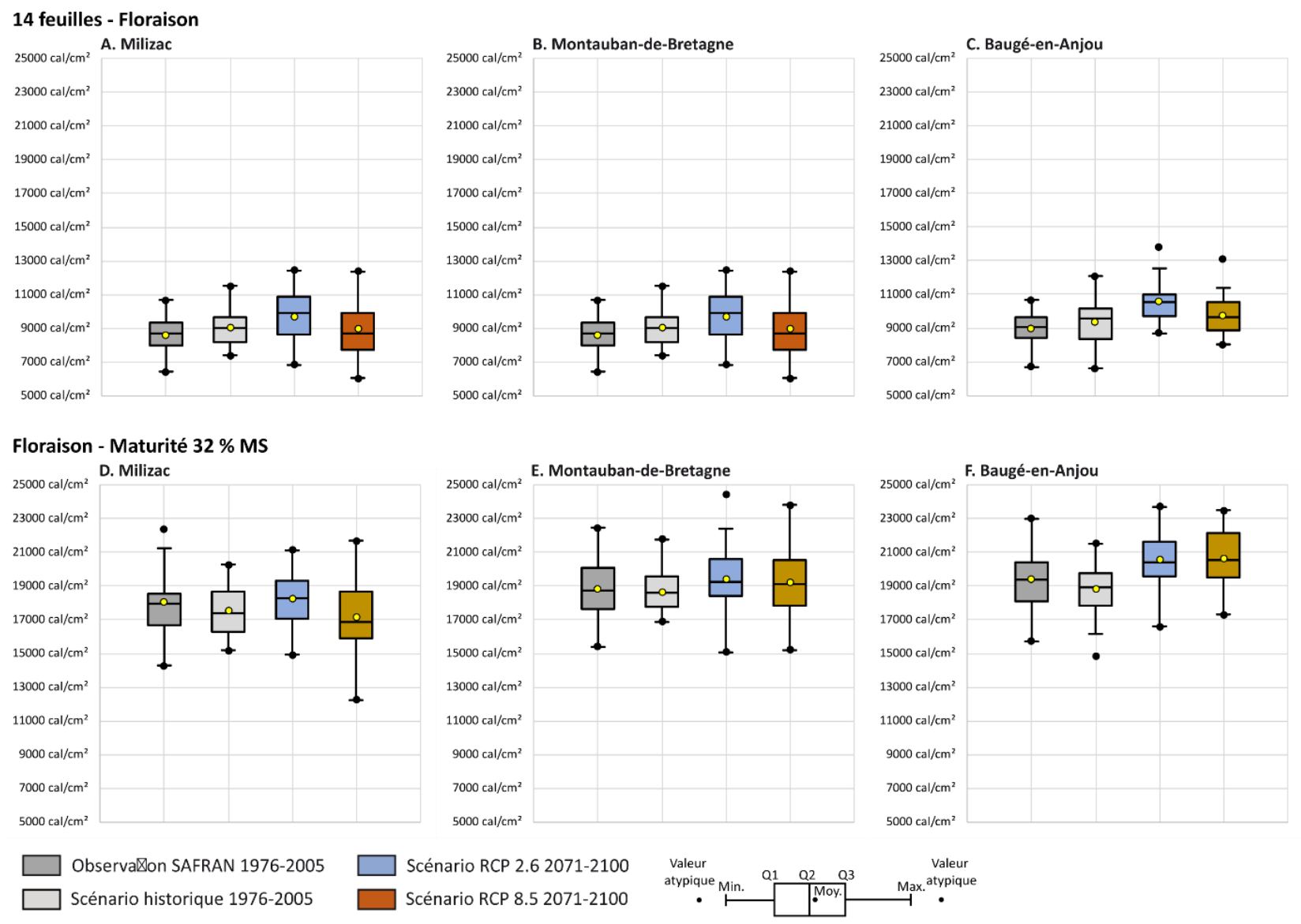

Figure 4. Rayonnement solaire cumulé sur les périodes 14 feuilles - floraison et floraison - maturité $32 \%$ MS du maïs fourrager pour la période de référence 1976-2005 et lointaine 2071-2100 selon deux scénarios RCP à Milizac (à gauche), Montauban-de-Bretagne (au centre) et Baugé-en-Anjou (à droite). Cumulative solar radiation over the periods 14 leaves - flowering and flowering - maturity 32\% DM of silage maize for the reference period 1976-2005 and 20712100 according to two RCP scenarios in Milizac (left), Montauban-de-Bretagne (center) and Baugé-en-Anjou (right).

\subsection{Quels effets d'une anticipation de la date de semis?}

Compte-tenu du réchauffement de l'atmosphère, les dates de semis pourraient être avancées si la température du sol le permet. Il conviendra toutefois de rester prudent aux excès en eau au début du cycle à Milizac (A) dans le cas d'un semis le $1^{\text {er }}$ avril pour le scénario RCP 2.6. En effet, la période semislevée serait exposée à $14,5 \mathrm{~mm}$ d'excès d'eau dans la parcelle (en déduisant la part allant à l'écoulement), contre $6,7 \mathrm{~mm}$ en période de référence pour un semis le $1^{\text {er }}$ mai. Le réchauffement quant à lui permettrait au maïs de ne pas rencontrer de gelées printanières au mois d'avril.

Les simulations effectuées pour des semis le 15 avril et le $1^{\text {er }}$ avril sur la période 2071-2100 ne permettent pas de réduire le déficit hydrique puisque l'essentiel du cycle de la plante s'effectue sur une période durant laquelle le sol est dépourvu en eau. Ce constat vaut pour les deux scénarios et sur les trois sites. En revanche, dans le cas du scénario RCP 2.6, la période 14 feuilles-floraison bénéficierait d'un rayonnement solaire cumulé d'environ 18 et $25 \%$ plus élevé (moyenne des trois sites) pour des semis respectivement les 15 avril et $1^{\mathrm{er}}$ avril par rapport à un maïs semé le $1^{\mathrm{er}}$ mai en période de référence. Dans le cas du scénario RCP 8.5, un semis le $1^{\text {er }}$ avril permettrait à Montaubande-Bretagne (B) et à Baugé-en-Anjou (C) de diminuer l'occurrence des très fortes chaleurs autour de la floraison qui apparaitraient alors couramment dès le mois de juin. Les occurrences seraient respectivement de 7 et 11 années sur 30 , contre 12 et 19 années sur 30 pour un semis le $1^{\text {er }}$ mai. 


\subsection{Quelle faisabilité d'une variété à la maturation tardive?}

Les variétés tardives ont un potentiel de rendement plus élevé que les variétés précoces. Pour considérer leur faisabilité, nous retenons que la maturité doit être intervenue avant le 15 novembre. Au-delà de cette date, les jours disponibles en base $6^{\circ} \mathrm{C}$ pour le développement se raréfient et les conditions climatiques peuvent être défavorables pour la plante (survenue des premières gelées) et pour les conditions d'ensilage (saturation en eau des sols).

En période de référence, le cycle n'aboutit que 1 et 2 années sur 3, respectivement à Milizac (A) et à Montauban-de-Bretagne (B). Même en anticipant la date de semis au 15 avril, les sommes de température restent insuffisantes. Le climat estival plus chaud de Baugé-en-Anjou (C) garantit en revanche sa faisabilité avec, d'après la modélisation, une date d'ensilage le 29 septembre. Dans ce cas, le cycle est plus long de 25 jours, soit un temps d'accumulation de biomasse et de rendement plus important. La plante profite d'un temps d'exposition plus long au rayonnement solaire. Les phases 14 feuilles-floraison et floraison-maturité $32 \%$ MS profiteraient respectivement de 14414 et $20739 \mathrm{cal} / \mathrm{cm}^{2}$ (tableau 3). Cependant, la plante cumule 20 et $15 \%$ de déficit hydrique de plus que la variété précoce pour les sols épais et mince (tableau 4).

Tableau 3. Rayonnement solaire cumulé en cal $/ \mathrm{cm}^{2}$ pour une variété tardive sur la période de référence 1976-2005 et la période future 2071-2100 selon deux scénarios et pour une variété précoce en période de référence 1976-2005 (date de semis le $1^{\mathrm{er}}$ mai) à Milizac, Montauban-de-Bretagne et Baugé-en-Anjou. Cumulative solar radiation in cal/ $\mathrm{cm}^{2}$ for a late variety over the reference period 1976-2005 and the future period 2071-2100 according to two scenarios and for an early variety in the reference period 1976-2005 (sowing date 1st May) in Milizac, Montauban-de-Bretagne and Baugé-en-Anjou.

\begin{tabular}{|c|c|c|c|c|}
\hline & \multicolumn{2}{|c|}{ 1976-2005 } & \multicolumn{2}{|c|}{ 2071-2100 } \\
\hline & Précoce & Tardive & $\begin{array}{l}\text { RCP } 2.6 \\
\text { Tardive }\end{array}$ & $\begin{array}{l}\text { RCP } 8.5 \\
\text { Tardive }\end{array}$ \\
\hline \multicolumn{5}{|c|}{14 feuilles - Floraison } \\
\hline Milizac & 9035 & - & 15630 & 14639 \\
\hline Montauban-de-Bretagne & 9368 & - & 16892 & 19376 \\
\hline Baugé-en-Anjou & 8997 & 14414 & 17419 & 16474 \\
\hline \multicolumn{5}{|c|}{ Floraison - Maturité $32 \%$ MS } \\
\hline Milizac & 17553 & - & 18316 & 17591 \\
\hline Montauban-de-Bretagne & 18644 & - & 20450 & 19376 \\
\hline Baugé-en-Anjou & 18812 & 20739 & 21389 & 21300 \\
\hline
\end{tabular}

Tableau 4. Déficit hydrique d'une variété tardive sur la période de référence 1976-2005 et la période future 2071-2100 selon deux scénarios RCP par rapport au déficit hydrique cumulé et pour une variété précoce en période de référence 1976-2005 (date de semis le $1^{\mathrm{er}}$ mai) à Milizac, Montauban-de-Bretagne et Baugé-en-Anjou. Water deficit of a late variety over the reference period 1976-2005 and the future period 2071-2100 according to two RCP scenarios in relation to the cumulative water deficit and for an early variety in the reference period 1976-2005 (sowing date 1st May) at Milizac, Montauban-de-Bretagne and Baugé-en-Anjou.

\begin{tabular}{|c|c|c|c|c|}
\hline & \multicolumn{2}{|c|}{ 1976-2005 } & \multicolumn{2}{|c|}{ 2071-2100 } \\
\hline & Précoce & Tardive & $\begin{array}{l}\text { RCP } 2.6 \\
\text { Tardive } \\
\end{array}$ & $\begin{array}{l}\text { RCP } 8.5 \\
\text { Tardive }\end{array}$ \\
\hline Milizac RUM 200 (mm) & 64 & - & 114 & 123 \\
\hline Milizac RUM 50 (mm) & 121 & - & 187 & 207 \\
\hline Montauban-de-Bretagne RUM 200 (mm) & 89 & - & 171 & 170 \\
\hline Montauban-de-Bretagne RUM 50 (mm) & 158 & - & 259 & 266 \\
\hline Baugé-en-Ajou RUM 200 (mm) & 112 & 135 & 215 & 213 \\
\hline Baugé-en-Anjou RUM 50 (mm) & 187 & 216 & 317 & 317 \\
\hline
\end{tabular}


À la fin $\mathrm{du} 21^{\mathrm{e}}$ siècle, le réchauffement modéré lié au scénario RCP 2.6 permettrait de rendre possible la culture d'une variété tardive sur les deux sites bretons. À Milizac (A), le cycle aboutirait 29 années sur 30 à une date d'ensilage le 21 octobre, soit une dizaine de jours plus tard qu'une variété précoce en période de référence. Certaines années, le cycle se terminerait au début du mois de novembre et il conviendrait dans ce cas de rester prudent face au risque d'épisodes de pluies susceptible de reporter les travaux de récolte plus tardivement dans l'automne, à des périodes peu favorables en raison de sols à nouveau exposés à l'excès d'eau. À Montauban (B), la maturité $32 \%$ MS aurait lieu le 28 septembre, et le 8 septembre à Baugé-en-Anjou (C). Dans le cas du scénario RCP 8.5 , la variété tardive arriverait à maturité plus tôt que la variété précoce en période de référence : le 14 septembre à Milizac (A), le 2 septembre à Montauban-de-Bretagne (B) et le 21 août à Baugéen-Anjou (C).

Le cycle étant plus long pour les variétés tardives, le maïs pourrait davantage valoriser le rayonnement solaire par rapport à une variété précoce en période historique. La phase phénologique 14 feuilles-floraison disposerait ainsi d'un cumul d'énergie 1,8 fois plus élevé pour les deux scénarios. En revanche, la fin du cycle ne disposerait de rayonnement solaire supplémentaire à Milizac (A) en raison d'une maturité intervenant plus tard dans la saison, moment de l'année durant lequel les jours sont plus courts et le soleil plus bas sur l'horizon. À Montauban-de-Bretagne (B), le cumul radiatif est en hausse de $10 \%$ pour le scénario RCP 2.6 et d'environ $14 \%$ pour les deux scénarios à Baugé-en-Anjou (C). Néanmoins, le cycle étant plus long et les sols dépourvus d'eau, le déficit hydrique serait beaucoup plus prononcé : 1,9 fois plus important pour les sols épais et 1,6 à 1,7 fois pour les sols minces (tableau 4). Les semis plus précoces, dès le début du mois d'avril, ne permettraient pas de réduire l'exposition aux sécheresses puisque le développement et la croissance se déroulent en plein été.

\section{Discussion et conclusion}

Les simulations bioclimatiques du maïs fourrager à partir des dernières données sorties du modèle régional ALADIN63_CNRM-CM5 permettent d'envisager les grandes tendances qui pourraient se dessiner d'ici la fin du $21^{\mathrm{e}}$ siècle pour la culture du maïs fourrage pour la région Grand Ouest.

D'ici 2100, le maïs cultivé pour l'ensilage effectuerait son cycle plus rapidement en raison de l'élévation de la température. Les temps d'accumulation de biomasse et d'élaboration du rendement seraient alors plus courts. La réduction de la durée des phases phénologiques ne permettrait pas de réduire l'exposition aux sécheresses estivales (plus précoces et plus intenses), ce qui constituerait un facteur limitant aux productions sans irrigation de cette plante qui consomme naturellement déjà beaucoup d'eau. Plus précisément, l'occurrence plus élevée de fortes températures autour de la floraison pourrait pénaliser la fertilité de la plante. A contrario, le cumul de rayonnement solaire global durant le cycle de vie de la plante resterait dans l'ensemble assez stable. L'élévation des températures permettrait la faisabilité de variétés tardives, plus productives. Néanmoins, en valorisant davantage de rayonnement solaire, elles seraient exposées à un déficit hydrique plus conséquent. Pour s'adapter, l'anticipation des semis ne permettrait pas de réduire l'exposition au manque d'eau. L'évitement n'étant pas envisageable, il conviendrait alors de développer des variétés tolérantes à ces derniers aléas au moment où des alternatives (luzerne, sorgho...) sont dès maintenant initiées.

Par ailleurs, cette étude démontre la transposabilité du modèle bioclimatique d'une culture à une autre. Néanmoins, pour préciser les conclusions fondées ici à partir de l'analyse de trois points, une chaîne de traitement pourrait être envisagée de façon à spatialiser les indicateurs phénoclimatiques sur l'ensemble du territoire couvert par la grille SAFRAN de résolution $8 \times 8 \mathrm{~km}$. Il serait alors possible de tenir compte des nuances intrarégionales et locales du climat du Grand Ouest de la France exposées par Dubreuil et al. (2013a), et même de proposer une approche au plus près des réalités du terrain en couplant ces entrées climatiques avec une connaissance de la réserve en eau des sols à méso-échelle (Beauvais $e t$ $a l ., 2019)$. Enfin, les calculs pourraient intégrer les données extraites des 11 autres couples GCM/RCM 
de l'expérience DRIAS-2020 (Soubeyroux et al., 2020) sous la forme d'une analyse multi-modèles. A titre d'exemple, pour le scénario RCP 8.5 à l'horizon 2071-2100, le modèle ALADIN63_CNRMCM5 simule une température moyenne supérieure de $+0,3^{\circ} \mathrm{C}$ à Milizac $(\mathrm{A}),+0,5^{\circ} \mathrm{C}$ à Montauban-deBretagne $(\mathrm{B})$ et $+0,7^{\circ} \mathrm{C}$ à Baugé-en-Anjou $(\mathrm{C})$ par rapport au modèle WRF381P_IPSL-CM5A.

\section{Références}

Allen R. G., Pereira L. S., Raes D., Smith M., 1998. Crop Evapotranspiration: Guidelines for Computing Crop Water Requirements. FAO Irrigation and Drainage Paper 56, FAO, Rome, 300 p. http://www.fao.org/3/X0490E/X0490E00.htm

Arvalis L'Institut du Végétal, 2021. Maïs variétés et interventions : région Bretagne. Choisir et décider : préconisations régionales 2021, $81 \mathrm{p}$.

Beauvais F., Cantat O., Madeline P., 2019a. Changement climatique et céréaliculture en Normandie : quelles perspectives pour 2100 ? Publications de l'Association Internationale de Climatologie, 32, 71-76. http://www.climato.be/aic/colloques/actes/Thessaloniki2019 _actes.pdf

Beauvais F., Cantat O., Madeline P., Le Gouée P., BrunelMuguet S., Mejkane M., 2019b. Quelles conséquences du changement climatique sur le blé tendre en Normandie aux horizons 2050 et 2100 ? Étude d'impact prospective à partir du modèle ALADIN-Climat. Climatologie, 16, 127-158 https://doi.org/10.4267/climatologie.1414

Beauvais F., Cantat O., Le Gouée P., Brunel-Muguet S., Madeline P., Gaillard H., Bataille M. P., Sallent A., Preux T., Medjkane M., 2020. Conséquences du changement climatique sur la culture du lin fibre en Normandie : étude d'impact prospective à partir des données CNRM-2014 et IPSL-2014. Actes du 32 ème Colloque de l'Association Internationale de Climatologie, Rennes, 97-102. http://www.climato.be/aic/colloques/actes/Rennes2020_actes.pdf

Bonhomme R., 2000. Bases and limits to using 'degree.day' units. European Journal of Agronomy, 13 (1), 1-10. https://doi.org/10.1016/S1161-0301(00)00058-7

Cantat O., Le Gouée P., Bensaid A. 2009. Le rôle de la topographie et des sols dans la modélisation spatiale d'échelles fines et des bilans hydriques en Normandie. Actes des journées de climatologie du CNFG de la commission Climat et Société, 81100. https://www6.rennes.inra.fr/climaster/content/download/3243/ 32894/version/1/file/CANTAT-et-al_CNFG-2009_ARTICLE.pdf

Cantat O., Le Gouée P., Bensaid A., Savouret E., 2010. Une méthode originale de spatialisation d'échelle fine des bilans hydriques. Publications de l'Association Internationale de Climatologie, 23, 101106. http://www.climato.be/aic/colloques/actes/rennes2010_actes.pdf

Carpentier B., Cabon G., 2011. Le maïs fourrage : élaboration du rendement et de la qualité, récolte et conservation. Fourrages, 205, 11-23.
Caubel J., Garcia De Cortazar-Atauri I., Launay M., De NobletDucoudre N., Huard F., Bertuzzi P., Graux A. I., 2015. Broadening the scope for ecoclimatic indicators to assess crop climate suitability according to ecophysiological, technical and quality criteria. Agricultural and Forest Meteorology, 207, 94106. http://dx.doi.org/10.1016/j.agrformet.2015.02.005

Caubel J., Garcia De Cortazar-Atauri I., Vivant A.-C., Launay M., De Noblet-Ducoudré N., 2018. Assessing future meteorological stresses for grain maize in France. Agriculturals Systems, 159, 237-247. 10.1016/j.agsy.2017.02.010.

Doorenbos J., Pruitt W.O., 1975. Guidelines for Predicting Crop Water Requirements. FAO Irrigation and Drainage Paper 24, FAO, Rome, 154 p.

Dubreuil V., 1994. La sécheresse dans la France de l'ouest : étude d'après les bilans hydriques et les données des satellites NOAA-AVHRR. Thèse de Doctorat, Université de Rennes 2, $381 \mathrm{p}$.

Dubreuil V., Lamy C., Planchon O., 2018. Les sécheresses à Rennes : Passé, présent et futur. Actes du colloque : Les risques naturels dans le contexte du changement climatique à Cluj (Roumanie), 15-21. https://halshs.archives-ouvertes.fr/halshs01825987v2/document

Dubreuil V., Planchon O., Lamy C., Bonnardot V., Quénol H., 2013a. Le changement climatique dans la France de l'Ouest : observation et tendances. In Merot P., Dubreuil V., Delahaye D., Desnos P. [Dir.], 2013, Changement climatique dans l'Ouest: Evaluation, impacts, perceptions, Presses Universitaires de Rennes, Rennes, 19-30.

Dubreuil V., Quénol H., Bigot S., Bonnardot V., Huard F., Déqué M., 2013b. Le climat de la France de l'Ouest au XXI ${ }^{\mathrm{e}}$ siècle : que disent les modèles? In Merot P., Dubreuil V., Delahaye D., Desnos P. [Dir.], 2013, Changement climatique dans l'Ouest: Evaluation, impacts, perceptions, Presses Universitaires de Rennes, Rennes, 57-72.

Gate P., 2009. Les raisons du plafonnement des rendements du blé en France. Perspectives Agricoles, 355, 6-12.

Gate P., Blondot A., Gouache D., Deudon O., Vignier L., 2008. Impacts du changement climatique sur la croissance et le développement du blé en France. Quelles solutions et quelles actions à développer? Oilseeds and fats Crops and Lipids Journal, 15, 5, 332-336

Gouache D., Le Bris X., Bogard M., Deudon O., Page C., Gate P., 2012. Evaluating agronomic adaptation options to increasing heat stress under climate change during wheat grain filling in France. European Journal of Agronomy, 39, 62-69. https://doi.org/10.1016/j.eja.2012.01.009

Hawkins E., Fricker T.-E., Challinor A.-J., Ferro C.-A.-T., Ho C-K-T., Osborne T.-M., 2013. Increasing influence of heat stress on French maize yields from the 1960s to the 2030s. Global Change Biology, 19, 937-947, 10.1111/gcb.12069.

Holzkämper A., Calanca P., Fuhrer J., 2011. Analyzing climate effects onagriculture in time and space. Procedia Environ. Sci., 3, 58-62. https://doi.org/10.1016/j.proenv.2011.02.011

Holzkämper A., Calanca P., Fuhrer J., 2013. Identifying climatic 
limitations to grain maize yield potentials using a suitability evaluation approach. Agricultural and Forest Meteorology, 168, 149-159. https://doi.org/10.1016/j.agrformet.2012.09.004

Holzkämper A., Fossati D., Hiltbrunner J., Fuhrer J., 2015. Spatial and temporal trends in agro-climatic limitations to production potentials for grain maize and winter wheat in Switzerland. Regional Environmental Change, 15, 109-122. https://doi.org/10.1007/s10113-014-0627-7

Merot P., Dubreuil V., Delahaye D., Desnos P., [Dir.] 2013. Changement climatique dans l'Ouest : Evaluation, impacts, perceptions. Presses Universitaires de Rennes, Rennes, 458 p.

Mkhabela M., Bullock P., Gervais M., Finlay G., Sapirstein H., 2010. Assessing indicators of agricultural drought impacts on spring wheat yield and quality on the Canadian prairies. Agricultural and Forest Meteorology, 150, 3, 399410. https://doi.org/10.1016/j.agrformet.2010.01.001

Lamy C. et Dubreuil V., 2013a. Impact potentiel du changement climatique sur les sécheresses pédologiques en Bretagne au 21 $1^{\text {ème }}$ siècle. Climatologie, 10, 107-121. http://lodel.irevues.inist.fr/climatologie/index.php?id=96

Lamy C., Cantat O., Le Gouée P., Dubreuil V., Bensaïd A., Lemercier B, Savouret E., 2013. Sécheresse et réserve en eau des sols. In Merot P., Dubreuil V., Delahaye D., Desnos P. [Dir.] 2013, Changement climatique dans l'Ouest: Evaluation, impacts, perceptions, Presses Universitaires de Rennes, Rennes, 19-30.

Le Gouée P., Cantat O, Bensaid A., Savouret E., 2010. La sensibilité des systèmes de production agricole en Normandie face au changement climatique. Publications de l'Association Internationale de Climatologie, 23, 333336. http://www.climato.be/aic/colloques/actes/rennes2010 _actes.pdf

Le Gouée P., Marie M., Cantat O., Bensaid A., 2010. Quand le géographe fait du sol une interface essentielle entre agriculture durable, société et environnement. Exemple de deux études de cas traitées en Basse-Normandie (France). $I S D A, \quad 28-30$ juin 2010, 10 p., https://hal.archivesouvertes.fr/hal-00521286/document

Quintana-Segui P., Le Moigne P., Durand Y., Martin E., Habets E., Baillon M., Canellas C., Franchisteguy L., Morel S., 2008. Analysis of near-surface atmospheric variables: Validation of the SAFRAN analysis over France. J. Appl. Meteor. Climatol., 47, 92-107. https://doi.org/10.1175/2007JAMC1636.1

Soubeyroux J.-M., Martin E., Franchistéguy L., Habets F., Noilhan J., Baillon M., Regimbeau F., Vidal J.-P., Le Moigne P., Morel S., 2008. L'application Safran - Isba - Modcou (SIM), un outil pour la production opérationnelle et les études hydrométéorologiques. La Météorologie, 8, 63, 40-45. $10.4267 / 2042 / 21890$

Soubeyroux J.-M., Bernus S., Corre L., Drouin A., Dubuisson B., Etchevers P., Viviane Gouget V., Josse P., Kerdoncuff M., Samacoits R., Tocquer F., 2020. Les nouvelles projections climatiques de référence DRIAS 2020 pour la métropole, MétéoFrance, 97 p., http://www.drias-climat.fr/document/rapportDRIAS-2020-red3-2.pdf

Thorntwaite C., Mather J., 1955. The water balance. Centerton, Climatology, 104 p.

Trnka M., Rötter R.-P., Ruiz-Ramos M., Kersebaum K.-C., Olesen J.-E., Zalud Z., Semenov M.-A., 2014. Adverse weather conditions for European wheat production will become more frequent with climate change. Nature Climate Change, 4, 637-643. https://doi.org/10.1038/nclimate2242

Turc L., 1961. Evaluation des besoins en eau d'irrigation. Evapotranspiration potentielle. Annales agronomiques, 12, 13-49.

Van Vuuren D., Edmonds J., Kainuma M., Riahi K., Thomson A., Hibbard K., Hurtt G. C., Kram T., Krey V., Lamarque J.F., Masui T., Meinshausen M., Nakicenovic N., Smith S. J., Rose S. K., 2011. The representative concentration pathways: an overview. Climate Change, 109, 5-31. 10.1007/s10584011-0148-z.

Verfaillie D., Déqué M., Morin S. et Lafaysse M., 2017. The method ADAMONT v1.0 for statistical adjustment of climate projections applicable to energy balance land surface models, Geosci. Model Dev., 10, 4257-4283, 10.5194/gmd-10-42572017.

Vidal J.-P., Martin E., Franchisteguy L., Baillon M., Soubeyroux J.-M., 2010. A 50-year high-resolution atmospheric reanalysis over France with the Safran system. International Journal of Climatology, 30, 16271644. 10.1002/joc. 2003. 